\title{
La colaboración científica en turismo: grupos y redes de investigación en Cataluña*
}

\author{
José Antonio Corral-Marfil \\ Universidad de Vic. Departamento de Economía y Empresa \\ jacorralmarfil@gmail.com

\section{Gemma Cànoves Valiente} \\ Universidad Autónoma de Barcelona. Departamento de Geografía \\ gemma.canoves@uab.es
}

\section{Resumen}

El objetivo del presente artículo es analizar la colaboración científica en el campo del turismo en Cataluña: caracterizar a los grupos de investigación; estudiar la colaboración de grupos, instituciones y países; explorar el efecto de la multidisciplinariedad y las revistas, e identificar investigadores clave. Se elaboró un catálogo de grupos y se analizó la producción entre 2000 y 2010 mediante el cálculo de indicadores bibliométricos y el análisis de redes sociales. Hubo cuatro tipos de grupos, entre los 20 detectados, los cuales colaboraron interna y externamente a partes iguales. La incidencia de la coautoría aumentó durante la década, aunque no la extensión. La colaboración fue mayor en las revistas más prestigiosas, pero no quedó claro que la multidisciplinariedad aumentara la colaboración. La red de coautorías estuvo poco cohesionada, es decir, tuvo muchos investigadores aislados y subgrupos desconectados. Y la colaboración nacional y la internacional fueron las que más crecieron, aunque la local fue la más frecuente.

Palabras clave: turismo; colaboración científica; grupos de investigación; análisis de redes sociales; Cataluña.

* Este artículo está basado en dos estudios financiados por el Observatorio de Turismo de la Generalidad de Cataluña: Aproximació a la recerca sobre turisme a Catalunya (exp. 249/09) y II Fase del mapa de coneixement del turisme des de la perspectiva de la recerca universitària (exp. 268/10).

El trabajo se ha realizado en el marco de los Estudios de Doctorado en Geografía de la Universidad Autónoma de Barcelona. 
Resum. La col.laboració cientifica en turisme: grups i xarxes d'investigació a Catalunya

L'objectiu del present article és analitzar la col-laboració científica en el camp del turisme a Catalunya: caracteritzar els grups d'investigació; estudiar la col-laboració de grups, institucions i països; explorar l'efecte de la multidisciplinarietat i les revistes, i identificar-ne investigadors clau. Es va elaborar un catàleg de grups i es va analitzar la producció entre 2000 i 2010 mitjançant el càlcul d'indicadors bibliomètrics i l'anàlisi de xarxes socials. Es van detectar 20 grups, els quals es van classificar en quatre tipus. Els grups van col-laborar de manera interna i externa a parts iguals. La col-laboració va ser més elevada en les revistes més prestigioses, però no va quedar clar que la multidisciplinarietat augmentés la col-laboració. La xarxa de coautories va ser poc cohesionada, és a dir, hi va haver molts investigadors aillats i subgrups desconnectats. I la col.laboració nacional i la internacional van ser les que van créixer més, tot i que la local va ser la més freqüent.

Paraules clau: turisme; col-laboració científica; grups d'investigació; anàlisi de xarxes socials; Catalunya.

Résumé. La collaboration scientifique en tourisme: groupes et réseaux de recherche en Catalogne

Il s'agit d'analyser le poids de la collaboration scientifique dans le domaine du tourisme en Catalogne: caractériser les groupes de recherche; étudier la collaboration apportée par les groupes, les institutions et les pays; rechercher l'effet de la pluridisciplinarité et des revues; et identifier les chercheurs-clé. Un catalogue des groupes a été élaboré et la production entre 2000 et 2010 a été analysée grâce au calcul d'indicateurs bibliométriques et à l'analyse des réseaux sociaux. Il en est ressorti quatre types de groupes qui, parmi les 20 détectés, ont apporté une collaboration interne et externe à parts égales. L'incidence de la co-création a augmenté pendant cette décade mais non pas son extension. La collaboration a été plus importante dans les revues les plus prestigieuses mais sans permettre cependant de pouvoir assurer que le rôle de la pluridisciplinarité a été facteur d'augmentation de la collaboration. Le réseau des co-auteurs a été peu uni: de nombreux chercheurs isolés et des sous-groupes non reliés. Ce sont les collaborations nationale et internationale qui ont enregistré la plus grande croissance mais la collaboration locale a été la plus fréquente.

Mots clé: tourisme; collaboration scientifique; groupes de recherche; analyse réseaux sociaux; Catalogne.

\section{Abstract. Scientific collaboration in tourism: research groups and networks in Catalonia}

The aim was to analyse research collaboration in the field of tourism in Catalonia: to describe the research groups; to study the collaboration between groups, organisations and countries; to explore the level of multidisciplinarity in relation with the type of journals; and to identify key researchers. We made a catalogue of groups and analysed research output between 2000 and 2010 by means of bibliometrics and social networks analysis. We found four types of groups among the 20 groups identified, collaborating internally and externally in equal measure. Incidence of co-authorship increased over the course of the decade, but not its extent. Collaboration was highest in the most prestigious journals, but it was not clear whether multidisciplinary studies correlated with higher collaboration. Co-authorship networks lacked cohesion: many researchers were working in isolation and disconnected subgroups. National and international collaboration increased the most, but local collaboration was the most frequent.

Keywords: tourism; research collaboration; research groups; social network analysis; Catalonia. 


\section{Sumario}
1. Introducción y objetivo
4. Resultados
2. Revisión de la literatura
5. Discusión y conclusiones
3. Metodología
Bibliografía

\section{Introducción y objetivo}

En paralelo al continuo crecimiento del turismo, la investigación turística ha ganado tamaño y reconocimiento en los últimos años. Ha emergido como un campo de estudio multidisciplinar y dinámico dentro de las ciencias sociales. Son indicadores de tal dinamismo la proliferación de revistas científicas, la defensa de tesis doctorales, la fundación de centros de investigación y la celebración de congresos (Racherla y Hu, 2010). En España, la academia turística también está creciendo, como muestran el aumento de la producción científica (Albacete y Fuentes, 2010) y la creación de estudios de grado, máster y doctorado (Ceballos et al., 2010). La geografía ha desempeñado un papel fundamental en esta expansión (Corral y Cànoves, 2013).

La colaboración científica ha aumentado durante las últimas décadas en todas las disciplinas (Glänzel y Schubert, 2004). El trabajo en equipo es una característica de la ciencia moderna, como lo son las elevadas necesidades de financiación (De Solla Price, 1966). La colaboración en las ciencias sociales es mucho menor que en las ciencias naturales y experimentales (Laband y Tollison, 2000), aunque es algo mayor que en las humanidades (Ardanuy, 2012). La colaboración está presente incluso en las ciencias «menos caras», como las matemáticas puras o la investigación teórica en ciencias sociales.

Por colaboración científica se entiende «la interacción entre dos o más científicos que tiene lugar dentro de un contexto social, la cual permite compartir significado y completar tareas con respecto a una meta superior mutuamente compartida» (Sonnenwald, 2007: 645). Se puede clasificar desde los siguientes puntos de vista: $a$ ) disciplinario (intradisciplinaria, interdisciplinaria, multidisciplinaria); b) geográfico (presencial, remota, internacional), y c) organizativo (universidad y empresa, ciencia y sociedad, universidad, industria y gobierno o "triple hélice»).

Beaver (2001) cita 18 motivos por los que los investigadores cooperan, entre los cuales, encontramos: acceder a conocimiento experto, equipamiento o recursos; obtener prestigio; ganar productividad; progresar más rápidamente; detectar errores más eficientemente; aprender; reducir el aislamiento; pasarlo bien, etc., pero, al mismo tiempo, la colaboración comporta mayores costes que el trabajo en solitario (Katz y Martin, 1997); por ejemplo: por gastos adicionales en desplazamientos, dietas y transporte de equipos; por inversión de tiempo en informar a los miembros del equipo y resolver discrepancias, o por el aumento de la burocracia y los costes administrativos.

El objetivo de este trabajo es analizar la colaboración científica de las universidades catalanas en el campo del turismo. Más específicamente, 
pretende: cuantificar la colaboración; descubrir diferencias entre revistas en cuanto a la colaboración; explorar la relación entre multidisciplinariedad y colaboración; visualizar la red social de coautorías y estudiar patrones de colaboración; identificar investigadores clave; describir la colaboración entre grupos, instituciones y países, e investigar sobre las características de los grupos de investigación existentes.

El análisis se circunscribe a la universidad, porque esta institución genera más del $85 \%$ de la producción científica sobre turismo en España (GonzálezAlbo et al., 2009), y a Cataluña, por el peso que tiene el turismo en la economía y en la ciencia de esta comunidad autónoma. En efecto, la historia del turismo en Cataluña se remonta al siglo XIX (Garay y Cànoves, 2010). Actualmente, es la segunda región de Europa más visitada por turistas extranjeros (Eurostat, 2011). El sector turístico representa alrededor del 11\% del PIB y también del empleo (Direcció General de Turisme, 2011). Y su geografía turística es diversa: tiene turismo litoral, de montaña, urbano y rural (Torres, 2010). Además, las universidades catalanas son autoras del $20 \%$ de la producción científica sobre turismo de España (González-Albo et al., 2009).

\section{Revisión de la literatura}

\subsection{El estudio de la investigación turistica}

Benckendorff (2009) propone dos ejes para clasificar las técnicas que se emplean para estudiar un campo de investigación. En primer lugar, distingue entre técnicas cualitativas y cuantitativas. La mayoría de las cuantitativas forman parte de la bibliometría. En segundo lugar, divide las técnicas entre valorativas y relacionales. Las valorativas persiguen comparar las contribuciones de autores, revistas, instituciones o trabajos académicos. Las relacionales buscan poner de manifiesto relaciones dentro de la investigación, como la estructura de los campos de conocimiento. El cruce entre ambos ejes da lugar a una tipología de cuatro categorías de técnicas.

Primero, las técnicas cualitativas valorativas consisten en elaborar rankings de revistas o autores a partir de opiniones de expertos. Por ejemplo, McKercher et al. (2006) jerarquizan 70 revistas de turismo y hostelería a partir de una encuesta a 505 académicos. Segundo, mediante técnicas cuantitativas valorativas, se analiza la producción científica para evaluar la contribución de instituciones, revistas o autores. Despiertan mucha controversia. A menudo, se basan en recuentos de artículos o citas y pueden considerar factores de impacto. Por ejemplo, Zhao y Ritchie (2007) identifican a los 57 investigadores más prolíficos en el campo del turismo, a partir del número de artículos que publicaron en ocho revistas entre 1985 y 2004.

Tercero, a través de técnicas cualitativas relacionales, se exploran tendencias y temas clave de la literatura, así como el uso de metodologías y técnicas estadísticas. Aquí, Benckendorff (2009) incluye las revisiones y las puestas al día, los mapas conceptuales, los análisis de contenido y los metaanálisis. Por 
ejemplo, Xiao y Smith (2006) identifican las 27 áreas temáticas principales en la investigación turística a lo largo de 30 años, a partir del análisis del índice de materias de la revista Annals of Tourism Research.

Y cuarto, a través de técnicas cuantitativas relacionales, se calculan indicadores bibliométricos para estudiar la investigación más allá de la productividad y los rankings. Incluyen los análisis de coocurrencias de términos (en el título o las palabras clave), de citas (a autores o artículos), cocitas, coautorías y redes sociales. Por ejemplo, Barrios et al. (2008) estudian la evolución de la producción sobre psicología del turismo entre 1990 y 2005, comprueban leyes bibliométricas y analizan la distribución de las citas y su relación con la colaboración. Constatan que los artículos con más de un autor reciben más citas que los firmados por un solo autor.

Pero la mayoría de estudios sólo contemplan revistas turísticas, cuando una parte considerable de la literatura se publica en revistas no turísticas. Hasta hace poco, ha habido pocas revistas turísticas con prestigio internacional (Chang y McAleer, 2012). Como afirma Sancho: «la escasez de revistas dedicadas al turismo, con validez y reconocimiento para la evaluación de la actividad investigadora, hace que las investigaciones en turismo queden dispersas en un abanico de revistas de diferentes disciplinas» (Sancho, 2006: 57).

El presente estudio analiza la colaboración científica en turismo cuantitativamente, y contempla revistas turísticas y no turísticas. Se enmarca en la cuarta categoría: la que analiza el contenido, la estructura y la evolución de la investigación con el fin de contribuir a su desarrollo.

\subsection{El estudio de la colaboración cientifica}

El proceso de la colaboración científica consta de cuatro etapas: establecimiento, formulación, mantenimiento y conclusión (Sonnenwald, 2007). En cada etapa, surgen factores que influyen sobre la colaboración. De entrada, factores científicos, políticos o socioeconómicos pueden impulsar o impedir su establecimiento. Después, durante la fase de formulación, se planifica el proyecto. Cuando se trabaja en colaboración, algunos factores deben detallarse más que cuando se trabaja en solitario, como los objetivos, las tareas, el liderazgo, la organización o la propiedad intelectual.

Una vez establecida la colaboración y comenzado el trabajo, hay que mantenerla durante un tiempo hasta alcanzar los objetivos. Suelen surgir contratiempos que pueden hacerla fracasar. Identificar y solucionar los problemas puede requerir modificar la estructura organizativa, las tareas, la comunicación, etc. Por último, idealmente, la colaboración concluye con éxito cuando se alcanzan los objetivos y se divulgan los resultados.

La coautoría representa la relación formal, tangible y directa que existe entre investigadores (Ye et al., 2011). Es el indicador más usado para cuantificar la colaboración (Benckendorff, 2010). Presenta ventajas: es invariable y verificable, de obtención fácil y barata, permite recolectar muestras grandes y no es intrusiva ni reactiva (Katz y Martin, 1997). Su inconveniente es que 
no refleja perfectamente la colaboración. Hay colaboraciones que no se plasman en coautorías. Y hay trabajos firmados en coautoría que no derivan de una estrecha colaboración entre los firmantes.

El aumento de la coautoría en las últimas décadas se debe a factores generales y específicos (Sonnenwald, 2007). Entre los generales, están la especialización, la división del trabajo científico, el aumento del número de investigadores y la mejora de los sistemas de comunicación. Entre los específicos, la naturaleza cuantitativa, la interdisciplinariedad y, en las ciencias naturales, el uso compartido de laboratorios y equipamientos muy costosos. En último término, el motivo para colaborar es aumentar la cantidad y la calidad de la producción. La colaboración aumenta la cantidad de trabajos que un investigador puede acometer. Y los artículos firmados en coautoría tienen mayor probabilidad de ser aceptados por las revistas (Acedo et al., 2006).

\subsection{El análisis de redes sociales}

El análisis de redes sociales se ocupa del estudio de las relaciones (amistades, transacciones, etc.) entre actores (personas, organizaciones, etc.) (Molina, 2004). Desde la investigación seminal de Newman (2001) sobre la estructura de las redes de colaboración científica, el uso de redes de coautorías ha acontecido una forma común de estudiar la colaboración científica.

Una red es un conjunto de relaciones (líneas o vínculos) entre una serie definida de elementos (nodos). En el caso de una red de coautorías, los nodos representan investigadores y las relaciones, coautorías. Es decir, dos investigadores están conectados si han publicado al menos un artículo conjuntamente. Las relaciones son recíprocas (no orientadas), puesto que si A firma con B, B firma con A. Y son valoradas, es decir, pueden tomar valores distintos de uno y cero (existencia o ausencia de relación). Los valores expresan el número de coautorías entre dos autores.

El gráfico 1 ilustra cómo construir una red de coautorías mediante un ejemplo. A partir de 5 artículos escritos por 10 investigadores, se elabora la matriz de relaciones con el programa Ucinet y se visualiza el grafo con el software Netdraw. La red se puede analizar a nivel macro y a nivel micro. Por ejemplo: la densidad, que mide la cohesión de la red, se calcula dividiendo el número de vínculos existentes entre el número de vínculos posibles. En este caso, hay 15 vínculos de los 45 posibles y, por tanto, la densidad es del 33\%. O, a nivel micro, el grado de un nodo (degree) es el número de nodos con los que está directamente conectado. Es una medida de su centralidad. En el ejemplo, el investigador con más colaboradores es Sáez, con 6.

\subsection{Los grupos de investigación}

Los grupos de investigación son una manifestación paradigmática de la colaboración científica. Constituyen la unidad micro más característica dentro de los tres niveles de organización de la ciencia (macro: ministerios, universidades; 


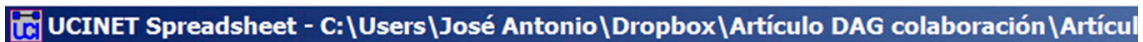

File Edit Transform Fill Labels Options Help

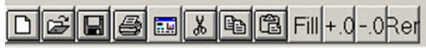

\begin{tabular}{|l|r|r|r|r|r|r|r|r|r|r|r|r|r|}
\hline \hline & Blasco & Coenders & Donaire & Espinet & Fluvià & Gali & Guia & Mundet & Prats & Sáez \\
\hline Blasco & 0 & 0 & 0 & 0 & 0 & 0 & 1 & 0 & 1 & 1 \\
\hline Coenders & 0 & 0 & 0 & 1 & 1 & 0 & 0 & 1 & 0 & 1 \\
\hline Donaire & 0 & 0 & 0 & 0 & 0 & 1 & 0 & 1 & 0 & 0 \\
\hline Espinet & 0 & 1 & 0 & 0 & 1 & 0 & 0 & 0 & 0 & 1 \\
\hline Fluviàn & 0 & 1 & 0 & 1 & 0 & 0 & 0 & 0 & 0 & 1 \\
\hline Galín & 0 & 0 & 1 & 0 & 0 & 0 & 0 & 0 & 0 & 0 \\
\hline Guia & 1 & 0 & 0 & 0 & 0 & 0 & 0 & 0 & 1 & 1 \\
\hline Mundet & 0 & 1 & 1 & 0 & 0 & 0 & 0 & 0 & 0 & 0 \\
\hline Prats & 1 & 0 & 0 & 0 & 0 & 0 & 1 & 0 & 0 & 1 \\
\hline Sánez & 1 & 1 & 0 & 1 & 1 & 0 & 1 & 0 & 1 & 0 \\
\hline
\end{tabular}

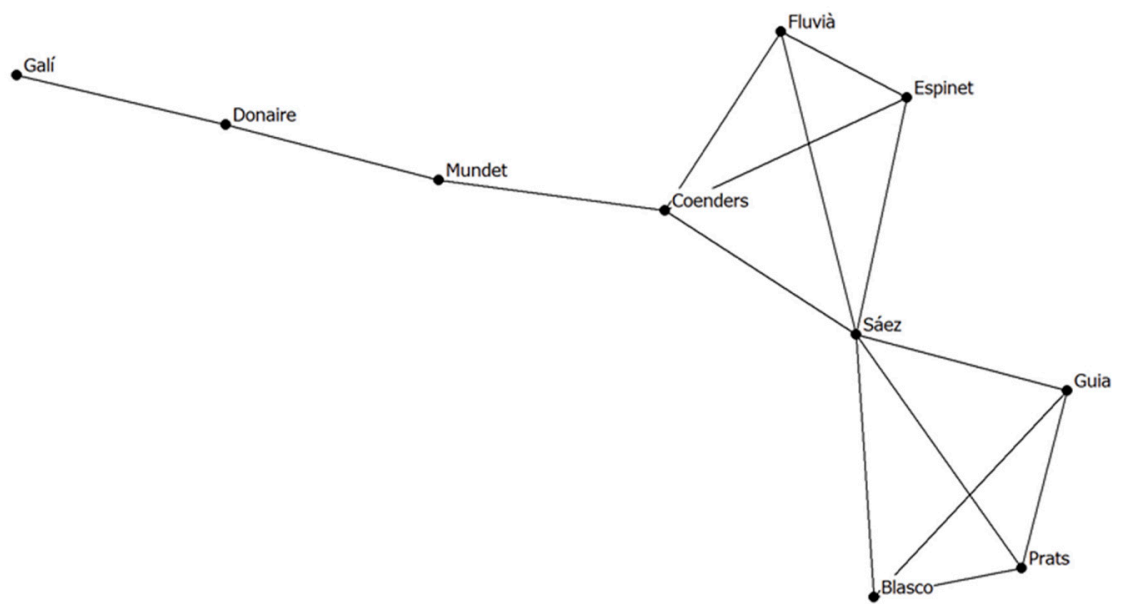

Blasco, Daniel; GuiA, Jaume Ò.; Prats, Lluís y SÁEZ, Marc (2009). «Clusters turísticos en Cataluña: Una propuesta de organización turística del territorio». RESTMA, 8-9, 77-97.

DonAIRE, José A. y MunDET, Lluís (2001). «Estrategias de reconversión turística de los municipios litorales catalanes». Papers de Turisme, 29, 51-65.

ESPINET, Josep M.; SÁEZ, Marc; CoEnders, Germà y FluVIÀ, Modest (2003). «Effect on prices of the attributes of holiday hotels: a hedonic prices approach». Tourism Economics, 9 (2), 165-177.

Galí, Núria y DonalRe, José A. (2006). «Visitors' Behavior in Heritage Cities: The Case of Girona». Journal of Travel Research, 44 (4), 442-448.

MUNDET, Lluís y COENDERS, Germà (2010). «Greenways: a sustainable leisure experience concept for both communities and tourists». Journal of Sustainable Tourism, 18 (5), 657-674.

Gráfico 1. Ejemplo del proceso de construcción de una red de coautorías.

Fuente: elaboración propia. 
meso: institutos, departamentos; micro: equipos, grupos). Un grupo de investigación es un "conjunto de dos o más personas que desarrollan su actividad científica en la misma unidad (departamento, laboratorio, etc.); con líneas de investigación, objetivos e intereses científicos comunes; que comparten tareas y recursos; publican conjuntamente de forma habitual; y disponen de cierta autonomía económica y de decisión» (Rey et al., 2008: 119).

Según Etzkowitz (2003), los grupos presentan cualidades que llevan a calificarlos de cuasi empresas, como, por ejemplo, la financiación de sus actividades en condiciones competitivas o los contactos con empresas y gobiernos para conseguir contratos de investigación y transferencia de conocimiento. Los grupos constituyen las células de la universidad emprendedora.

Sin embargo, no existe consenso acerca del concepto de grupo de investigación. Por ejemplo, la Ley Orgánica de Universidades menciona los grupos, pero no los define, por lo que cada universidad aplica sus propios criterios para reconocer a sus grupos. Rey et al. (2008) contrastan los conceptos de grupo y equipo de investigación. El equipo es una asociación ad hoc para desarrollar un proyecto concreto y, por tanto, es temporal. El grupo es más estable, porque está vinculado a líneas de investigación que no tienen por qué tener una duración determinada. Los miembros de los grupos suelen pertenecer a la misma organización, mientras que los de los equipos suelen ser más heterogéneos en cuanto a adscripción, localización y nacionalidad.

El estudio de los grupos de investigación se aborda desde dos enfoques metodológicos (Cohen, 1991). Los estudios basados en las entradas (inputbased studies) identifican los grupos a partir de unidades organizativas existentes, como laboratorios, institutos o grupos formales, mientras que los estudios basados en los resultados (output-based studies) los definen a partir de su producción, mediante el análisis de frecuencias de coautorías. Rey et al. (2008) denominan los grupos resultantes, respectivamente: «grupos estructurales» $y$ «grupos funcionales».

Referido a la geografía del turismo, Fernández et al. (2010) describen los centros, los grupos y las líneas de investigación de las comunidades autónomas españolas. Sin embargo, en el campo del turismo en general, ningún trabajo ha analizado los grupos de investigación siguiendo el enfoque estructural hasta donde conocen los autores. Un objetivo de este estudio es identificar y analizar los grupos de investigación turística formalmente constituidos en las universidades catalanas. Además, pretende descubrir hasta qué punto son unidades estructurales o funcionales.

\subsection{El estudio de la colaboración en el campo del turismo}

Entre los pocos trabajos que estudian la colaboración científica en turismo (Racherla y Hu, 2010), destacan tres: Ye et al. (2013), Benckendorff (2010) y Racherla y Hu (2010). Los tres analizan la colaboración a partir de coautorías de artículos y los tres aplican el análisis de redes sociales.

Ye et al. (2012) investigan la colaboración entre académicos. Construyen la red social de coautorías a partir de los artículos publicados en seis importantes 
revistas de turismo y hostelería entre 1991 y 2010 . A nivel macro, muestran que la red es de "mundo pequeño», esto es, una red en la que la mayoría de nodos tienen pocos vecinos $\mathrm{y}$, sin embargo, en pocos pasos pueden alcanzar cualquier otro nodo (Molina, 2004). También comparan la red con la de otras disciplinas. A nivel micro, identifican nodos centrales que tienen muchas conexiones o enlazan subgrupos. Además, exploran la relación entre colaboración y productividad científica.

Benckendorff (2010) investiga la colaboración de individuos, instituciones y países. Estudia las coautorías de artículos publicados por investigadores australianos y neozelandeses en 18 revistas turísticas, entre 1999 y 2008. Analiza la incidencia, la extensión y la evolución de la coautoría; explora las pautas de colaboración a través del análisis de redes sociales, y muestra el aumento de la colaboración individual e internacional. La red de coautorías está dispersa, tiene muchos autores y grupos desconectados, y está dominada por un grupo o componente principal.

Racherla y Hu (2010) estudian los patrones de colaboración entre académicos del turismo mediante el análisis de redes sociales, identifican investigadores clave y presentan la evolución de la red de coautorías. Analizan los artículos publicados entre 1996 y 2005 en Annals of Tourism Research, Journal of Travel Research y Tourism Management. Constatan un aumento de la colaboración, una concentración de la colaboración en pocos autores y una baja cohesión de la red. Además, analizan la relación entre centralidad y productividad.

En España, casi no se ha estudiado la colaboración científica en el campo del turismo. Sánchez y Marín (2003) analizan los artículos publicados en las revistas Papers de Turisme y Estudios Turísticos entre 1996 y 2001. Miden la incidencia y la extensión de la coautoría teniendo en cuenta las áreas de la economía de la empresa, la nacionalidad de los autores y las universidades, así como el enfoque empírico o conceptual. Hernández et al. (2011) y Moreno y Picazo (2012) tocan tangencialmente el tema de la colaboración. Calculan la distribución del número de autores por artículo.

González-Albo et al. (2009) analizan la producción sobre turismo en España. Mediante una estrategia de búsqueda basada en palabras clave, recogen los documentos publicados en revistas turísticas y no turísticas entre 1998 y 2008. Dividen el estudio según la fuente de los datos: la base de datos internacional Web of Science y la española ISOC. Describen la evolución del número medio de autores, así como la colaboración entre centros, entre comunidades autónomas y entre España y otros países. Emplean limitadamente el análisis de redes sociales.

El presente estudio extiende estos análisis mediante el cálculo de indicadores de colaboración no relacionales, el análisis de redes sociales y el estudio de los grupos de investigación. En resumen, trata de responder a las siguientes preguntas:

- Medida, evolución y determinantes de la colaboración: ¿cuánto se colaboró? ¿Influyen las revistas, su indexación y la multidisciplinariedad sobre la colaboración? 
- Red de colaboración entre investigadores individuales: ¿cómo es la red de coautorías? ¿Quiénes son los investigadores clave?

- Colaboración institucional: ¿¿cuánto colaboran las instituciones? ¿Cómo es la red de colaboración entre instituciones?

- Colaboración territorial: ¿con qué comunidades autónomas y países colaboró Cataluña? ¿Cómo evolucionó la colaboración local, doméstica e internacional?

- Grupos formales de investigación turística: ¿qué grupos hay constituidos en las universidades catalanas? ¿Qué características presentan?

- Colaboración de los grupos: ¿son unidades funcionales o estructurales? ¿Cuánto colaboran entre sí?

\section{Metodología}

Se estudió la colaboración científica en el campo del turismo en Cataluña a través de un análisis bibliométrico de la producción científica y a través de la elaboración de un catálogo de grupos de investigación.

El estudio bibliométrico consistió, en primer lugar, en recolectar los artículos sobre turismo firmados por autores afiliados a universidades catalanas ${ }^{1}$, publicados entre 2000 y 2010. Solo se recolectaron artículos de investigación, no reseñas u otros tipos de documentos. Las fuentes fueron las bases de datos bibliográficas ISOC, ICYT, Scopus y CAB Direct. Las búsquedas en ISOC e ICYT permitieron cubrir los artículos publicados en revistas españolas. Con la búsqueda en Scopus, se cubrieron las principales revistas extranjeras. Y la búsqueda en $\mathrm{CAB}$ Direct permitió detectar artículos de revistas extranjeras no indexadas por Scopus. En CAB Direct está integrada la base de datos Leisure Tourism, cuya cobertura del campo del turismo es más amplia que la de Scopus.

La estrategia de búsqueda en las bases de datos ISOC, ICYT y Scopus se basó en una lista de términos afines al turismo (González-Albo et al., 2009; Corral y Cànoves, 2013), mientras que en CAB Direct se buscó dentro de los códigos temáticos Tourism and Travel y Leisure, Recreation and Tourism Economics. De esta forma, se cubrieron la mayoría de revistas nacionales e internacionales, turísticas y no turísticas.

A continuación, se depuraron los resultados de las búsquedas para eliminar duplicados y artículos no referidos al turismo. Como resultado, se obtuvieron 429 artículos, los cuales se grabaron con el gestor de referencias bibliográficas Refworks.

1. En Cataluña, hay doce universidades. Siete públicas: Universidad Autónoma de Barcelona (UAB), Universidad de Barcelona (UB), Universidad de Gerona (UdG), Universidad de Lérida (UdL), Universidad Politécnica de Cataluña (UPC), Universidad Pompeu Fabra (UPF) y Universidad Rovira i Virgili (URV); cuatro privadas: Universidad Abat Oliba (UAO-CEU), Universidad de Vic (UVic), Universidad Internacional de Cataluña (UIC) y Universidad Ramon Llull (URL), y una virtual: Universidad Abierta de Cataluña (UOC). 
Después, de cada artículo, se codificaron los atributos: año de publicación, autores, afiliación de los autores (grupo de investigación, universidad ${ }^{2}$, comunidad autónoma, país), revista, indexación de la revista (ISI ${ }^{3}$, Scopus), nacionalidad de la revista y disciplinas científicas del artículo y de la revista. Las disciplinas de las revistas indexadas en Scopus se obtuvieron a partir de los All Science Classification Codes. Las disciplinas de las revistas españolas se obtuvieron del sistema de clasificación de disciplinas de ISOC. Dado que, de ICYT, solo se recolectaron 11 artículos, estos se agruparon en una única disciplina llamada "Ciencia y tecnología».

Antes de efectuar el recuento de autores, se normalizaron sus nombres completos para evitar duplicidades. El sexo de los autores se asignó a partir de sus nombres. El recuento dio como resultado 508 autores diferentes, los cuales estamparon las 884 firmas de los 429 artículos. Al computar el número de artículos de autores, grupos, instituciones y territorios, se siguió el método del conteo de casos (instance counting method), según el cual un artículo firmado por dos autores otorga un punto (firma, autoría) a cada autor, no medio punto a cada autor (Severt et al., 2009).

Los artículos se analizaron mediante el cálculo de indicadores bibliométricos no relacionales y mediante el análisis de redes sociales. Al cuantificar la colaboración y su evolución, se distinguió entre la incidencia de la coautoría (proporción de artículos con más de un autor) y la extensión (número medio de autores de los artículos con más de un autor) (Laband y Tollison, 2000). Estos indicadores de la colaboración se cruzaron con indicadores del tipo de revista (indexación, nacionalidad) y de la multidisciplinariedad para explorar la influencia de estas variables sobre la colaboración.

Para el análisis de redes sociales, con la ayuda de una hoja de cálculo, se construyó la matriz de autorías y coautorías de los 508 autores diferentes de los 429 artículos. También se creó una matriz de atributos de los autores a partir de sus afiliaciones. Después, se exportaron las matrices al programa Ucinet 6 y se calcularon medidas de centralidad de los autores (rango, intermediación, cercanía) y de la estructura de la red (densidad, componente principal, centralidad media). Con el programa Netdraw, se representaron gráficamente las redes de coautorías.

Por otra parte, la elaboración del catálogo de grupos de investigación partió de un encargo del Observatorio de Turismo de Cataluña (exp. 249/09). El primer paso fue identificar grupos que investigaran sobre turismo. Se buscaron grupos con los términos turismo o turistico en su nombre en el listado de grupos

2. De los coautores afiliados a organismos públicos, se registró el departamento o centro, mientras que las empresas privadas se registraron al nivel organizativo más general (empresa o matriz).

3. Institute for Scientific Information, actualmente perteneciente a Thomson Reuters. La indexación se hace en tres listas: SCI (ciencias puras), SSCI (ciencias sociales) y AHCI (artes y humanidades) (Barriocanal et al., 2007), accesibles a través del servicio de bases de datos Web of Knowledge. 
reconocidos por la AGAUR ${ }^{4}$. También se buscaron grupos con líneas de investigación o producción sobre turismo en los webs de las universidades catalanas. Así, se detectaron 20 grupos. En segundo lugar, se solicitó información a los líderes de los 20 grupos sobre la misión, los miembros, las líneas de investigación y la producción científica. Y, en tercer lugar, se analizó la información, con el fin de caracterizar a los grupos.

A pesar de que se solicitó la producción científica a los grupos de investigación (y estos la suministraron muy amablemente), todos los indicadores bibliométricos y de redes sociales, que se presentan a continuación en la sección «Resultados», se calcularon a partir de los 429 artículos recopilados en el análisis bibliométrico, y no a partir de la producción suministrada por los grupos. Así se consiguió una homogeneidad en el criterio de selección de artículos y en el intervalo temporal considerado.

\section{Resultados}

\subsection{Incidencia y extensión de la coautoría}

De los 429 artículos que se publicaron a lo largo del decenio, 257 se realizaron en colaboración y 172, en solitario, es decir, la incidencia de la coautoría fue del 59,9\%. Del primer al segundo lustro, la incidencia aumentó del $51 \%$ al $65 \%$. El gráfico 2 muestra la evolución del número anual de artículos escritos en solitario y en colaboración.

El número de autores más frecuente en los trabajos escritos en colaboración fue dos. El 49\% de los artículos fueron firmados por dos o tres autores, y sólo

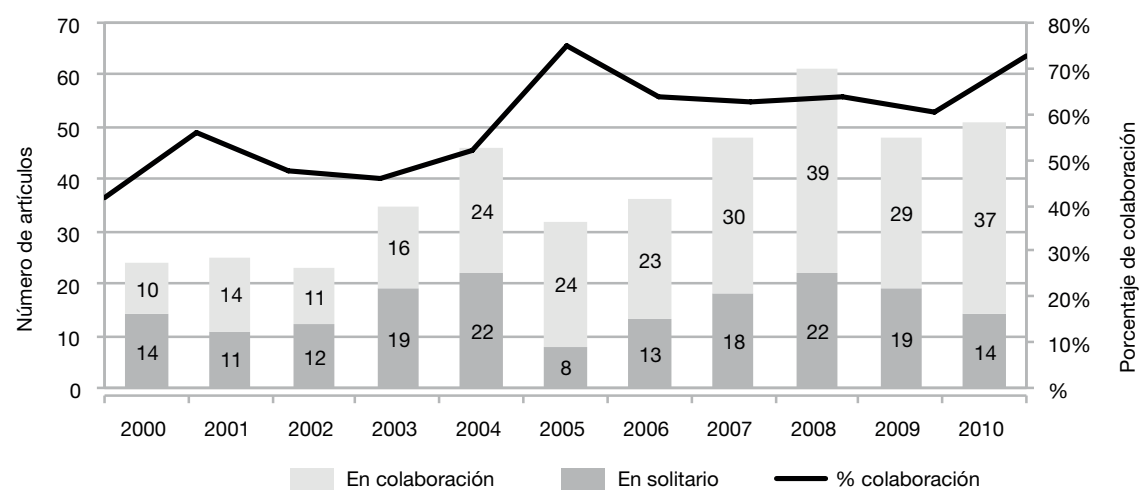

Gráfico 2. Evolución del número de artículos escritos en solitario y en colaboración.

Fuente: elaboración propia.

4. AGAUR: Agencia de Gestión de Ayudas Universitarias y de Investigación, dependiente de la Generalidad de Cataluña (en catalán: Agència de Gestió d'Ajuts Universitaris i de Recerca). 
el $4 \%$ por más de cuatro. La extensión de la coautoría, es decir, el número medio de autores de los artículos con más de un autor, fue de 2,77. La extensión disminuyó durante la década: de 2,94 autores por artículo en el primer lustro bajó a 2,69 en el segundo.

El 35,6\% de las firmas fueron de mujeres y el $64,4 \%$, de hombres. No hubo diferencias significativas entre los sexos en la incidencia de la coautoría. O sea, la proporción de artículos escritos en solitario fue casi igual entre las mujeres que entre los hombres. De los 152 artículos firmados por dos autores, el 12,5\% los escribieron dos mujeres; el 45,4\%, dos hombres, y el $42,1 \%$, un hombre y una mujer.

La tabla 1 muestra la incidencia y la extensión de la coautoría en las revistas con más de cuatro artículos. Aparentemente, hubo diferencias entre las revistas.

Los gráficos 3 y 4 muestran la incidencia de la coautoría según la indexación de las revistas. La incidencia fue significativamente mayor en las revistas indexadas al 95\% de confianza. También la extensión de la coautoría fue significativamente mayor en las revistas indexadas. La extensión en revistas ISI fue de 3,16 firmas por artículo, frente a las 2,61 en revistas no ISI. La extensión en revistas Scopus fue 3,07 firmas por artículo, frente a las 2,55 en revistas no Scopus.

Con el fin de explorar el efecto de la multidisciplinariedad sobre la colaboración, se comparó el grado de colaboración (extensión e incidencia de la coautoría)

Tabla 1. Incidencia y extensión de la colaboración en las revistas con más artículos

\begin{tabular}{|c|c|c|c|c|c|c|}
\hline Revista & Artículos & $\begin{array}{c}\% \text { de } \\
\text { artículos }\end{array}$ & $\begin{array}{c}\% \text { de } \\
\text { Incidencia }\end{array}$ & Extensión & Moda & Máximo \\
\hline Estudios Turísticos & 22 & 5,1 & 50 & 2,55 & 1 & 4 \\
\hline Estudis de Turisme de Catalunya & 18 & 4,2 & 22 & 2,25 & 1 & 3 \\
\hline Cuadernos de Turismo & 16 & 3,7 & 75 & 2,42 & 2 & 4 \\
\hline PASOS & 16 & 3,7 & 56 & 2,11 & 2 & 3 \\
\hline Tourism Management & 13 & 3,0 & 76 & 2,60 & 2 & 5 \\
\hline Documents d'Anàlisi Geogràfica & 11 & 2,6 & 63 & 2,00 & 2 & 2 \\
\hline Papers de Turisme & 11 & 2,6 & 63 & 2,14 & 2 & 3 \\
\hline Scripta Nova & 11 & 2,6 & 18 & 2,00 & 1 & 2 \\
\hline Boletín de la AGE & 9 & 2,1 & 66 & 2,50 & $1,2,3$ & 3 \\
\hline Íber & 9 & 2,1 & 22 & 2,00 & 1 & 2 \\
\hline Tourism Economics & 9 & 2,1 & 100 & 2,89 & 2 & 5 \\
\hline Revista de Análisis Turístico & 7 & 1,6 & 71 & 2,80 & 2 & 6 \\
\hline Cuadernos Geográficos & 6 & 1,4 & 66 & 2,75 & 1,2 & 4 \\
\hline $\begin{array}{l}\text { Annals of Tourism Research } \\
\text { en español }\end{array}$ & 5 & 1,2 & 80 & 4,00 & 5 & 5 \\
\hline Investigaciones Geográficas & 5 & 1,2 & 60 & 2,67 & 1,3 & 3 \\
\hline $\begin{array}{l}\text { Revista Aragonesa de Admón. } \\
\text { Pública }\end{array}$ & 5 & 1,2 & 100 & 2,40 & 2 & 3 \\
\hline Tourism Review & 5 & 1,2 & 100 & 2,20 & 2 & 3 \\
\hline 181 revistas restantes & 251 & 58,5 & 60 & 2,97 & 1 & 11 \\
\hline Total & 429 & 100,0 & 60 & 2,77 & 1 & 11 \\
\hline
\end{tabular}

Fuente: elaboración propia. 

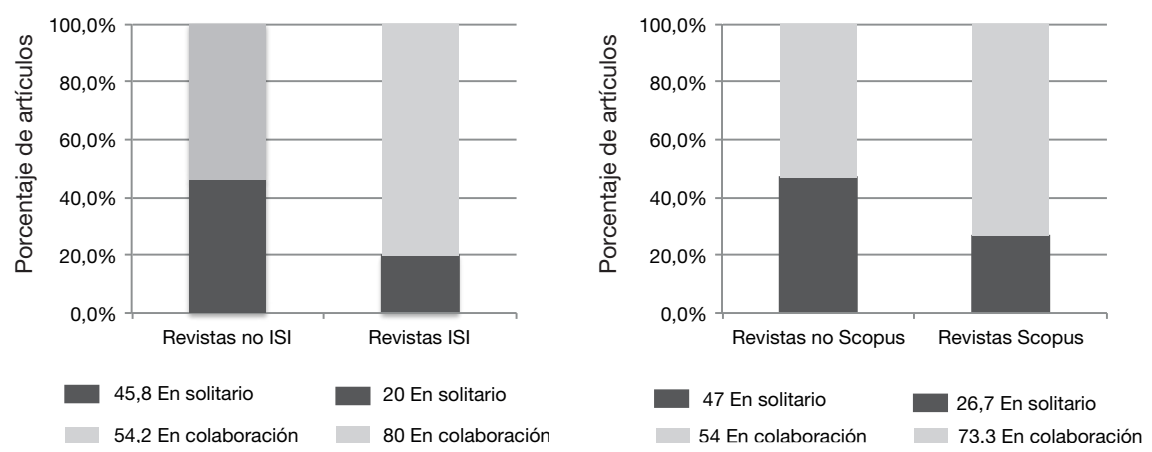

Gráficos 3 y 4 . Porcentajes de colaboración según indexación de las revistas.

Fuente: elaboración propia.

en los artículos unidisciplinares con el grado de colaboración en los artículos multidisciplinares (tabla 2). La comparación se realizó midiendo la multidisciplinariedad a través de tres indicadores alternativos, en tres subconjuntos distintos de artículos, respectivamente. Los tres indicadores y subconjuntos fueron: el número de disciplinas de los artículos, para los artículos publicados en revistas españolas; el número de disciplinas de las revistas, también para los artículos publicados en revistas españolas, y el número de disciplinas de las revistas, para los artículos publicados en revistas Scopus, españolas y extranjeras.

Referente a la incidencia de la coautoría, los resultados fueron contradictorios. En las revistas españolas, fue menor en los artículos multidisciplinares que en los unidisciplinares, en contra de lo esperado, mientras que en las revistas Scopus sí aumentó la colaboración con la multidisciplinariedad, de acuerdo con lo esperado. Respecto a la extensión de la coautoría, los resultados fueron más coherentes: en las tres medidas fue mayor en los artículos multidisciplinares que en los unidisciplinares. Si bien la diferencia fue pequeña y no significativa.

Tabla 2. Efecto de la multidisciplinariedad sobre la incidencia y la extensión de la coautoría

\begin{tabular}{|c|c|c|c|c|c|c|}
\hline \multirow[b]{3}{*}{ Número de disciplinas } & \multicolumn{4}{|c|}{ Revistas españolas } & \multirow{2}{*}{\multicolumn{2}{|c|}{$\begin{array}{c}\text { Revistas Scopus } \\
\text { Disciplinas de las revistas }\end{array}$}} \\
\hline & \multicolumn{2}{|c|}{ Disciplinas de los artículos ${ }^{1}$} & \multicolumn{2}{|c|}{ Disciplinas de las revistas ${ }^{2}$} & & \\
\hline & Incidencia & Extensión & Incidencia & Extensión & Incidencia & Extensión \\
\hline Una disciplina & $54,1 \%$ & 2,52 & $55,4 \%$ & 2,60 & $65,9 \%$ & 2,85 \\
\hline Más de una disciplina & $53,8 \%$ & 2,72 & $32,5 \%$ & 2,69 & $76,2 \%$ & 3,15 \\
\hline Valor prueba & $0,002^{\mathrm{a}}$ & $-0,978^{b}$ & $7,334^{a, *}$ & $-0,295^{b}$ & $1,609^{a}$ & $-0,790^{\mathrm{b}}$ \\
\hline
\end{tabular}

1. Se publicaron 307 artículos en revistas españolas, pero no se dispuso de las disciplinas de 53 artículos. Por ello, este análisis se basa en 254 artículos.

2. Referido a los 307 artículos publicados en revistas españolas.

3. Referido a los 146 artículos publicados en revistas indexadas en Scopus, españolas y extranjeras.

a) Chi-cuadrado de Pearson

b) T para igualdad de medias

${ }^{*} p \leq 0,05$

Fuente: elaboración propia. 


\subsection{Red de coautores}

El gráfico 5 muestra la red de coautorías. Los nodos representan autores y los vínculos, coautorías. El tamaño de los nodos es proporcional al número

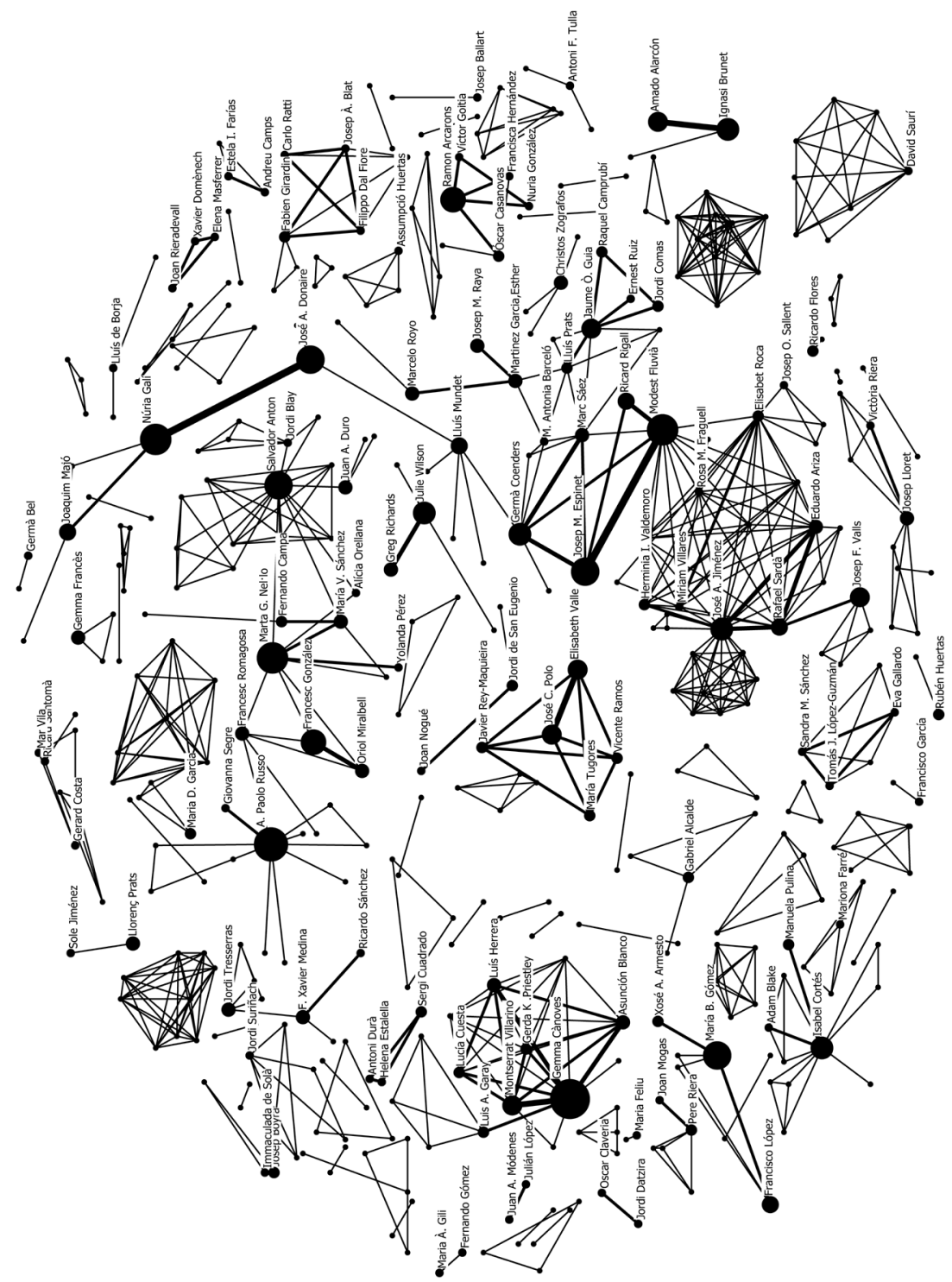

Gráfico 5. Red de colaboración entre autores.

Fuente: elaboración propia. 
de artículos de los autores. El grosor de los vínculos es proporcional al número de coautorías; una línea más gruesa entre dos autores indica que han publicado más artículos en coautoría. No se muestran los nodos aislados, sino sólo el nombre de los autores con más de un artículo.

Fue una red poco cohesionada, con multitud de nodos y subgrupos desconectados. La densidad fue 0,0055, es decir, sólo tuvieron lugar el 0,5\% de los vínculos posibles. Así pues, fue poco probable que un autor publicara con muchos otros autores.

Un componente es un conjunto de nodos que están conectados entre sí, directa o indirectamente, es decir, dos nodos pertenecen al mismo componente si existe un camino que los une. En la red, hay 171 componentes. El componente mayor contiene 61 nodos (12\% de los nodos) y el segundo mayor, 40 nodos (7,9\%). El resto de componentes tiene menos de 15 nodos $(3 \%)$.

Las medidas de centralidad permiten conocer la posición de los nodos en la red (Freeman, 1978-1979). La tabla 3 muestra el grado nodal (degree) o rango, el grado de intermediación (betweenness) y la cercanía (closeness) de los autores con mayores valores en estas medidas. El rango de un nodo es el número de

Tabla 3. Medidas de centralidad de los autores

\begin{tabular}{|c|c|c|c|c|c|c|c|c|c|}
\hline \multicolumn{2}{|c|}{ Autor Colaboradores } & \multicolumn{2}{|c|}{ Colaboraciones } & \multicolumn{2}{|l|}{ Intermediación } & \multirow{2}{*}{$\begin{array}{l}\text { nCercanía } \\
\text { M. Fluvià }\end{array}$} & \multicolumn{3}{|c|}{ Artículos } \\
\hline J. A. Jiménez & 23 & J. A. Jiménez & 31 & M. Fluvià & 909 & & 0,22314 & G. Cànoves & 15 \\
\hline S. Anton & 16 & G. Cànoves & 28 & G. Coenders & 631 & J.A. Jiménez & 0,22312 & A. P. Russo & 13 \\
\hline M. Fluvià & 14 & M. Fluvià & 26 & J.A. Jiménez & 590 & G. Coenders & 0,22312 & N. Galí & 12 \\
\hline E. Roca & 14 & J. M. Espinet & 19 & S. Anton & 501 & M. Sáez & 0,22312 & M. Fluvià & 11 \\
\hline A. P. Russo & 11 & S. Anton & 16 & L. Mundet & 480 & J. M. Espinet & 0,22311 & M. G. Nel·lo & 11 \\
\hline M. Villares & 11 & G. Coenders & 16 & M. Sáez & 456 & E. Roca & 0,22311 & J. M. Espinet & 10 \\
\hline R. M. Fraguell & 11 & L. Herrera & 16 & M. G. Nel·lo & 394 & M. Villares & 0,22311 & S. Anton & 10 \\
\hline G. Cànoves & 10 & J. C. Polo & 16 & F. Romagosa & 335 & H. Valdemoro & 0,22311 & J.A. Donaire & 10 \\
\hline I. Cortés & 10 & E. Roca & 15 & A. P. Russo & 333 & R. M. Fraguell & 0,22311 & M. B. Gómez & 10 \\
\hline H. Valdemoro & 10 & A. Blanco & 14 & J.A. Donaire & 279 & R. Rigall & 0,22308 & R. Arcarons & 9 \\
\hline M. Sáez & 8 & I. Cortés & 13 & E. Roca & 199 & L. Mundet & 0,22308 & F. González & 9 \\
\hline M. G. Nel·lo & 8 & M. Sáez & 13 & J. Ò. Guia & 196 & M. A. Barceló & 0,22308 & J. A. Jiménez & 8 \\
\hline G. Coenders & 7 & J. Ò. Guia & 13 & E. Martinez & 173 & J. Ò. Guia & 0,22307 & G. Coenders & 8 \\
\hline L. Herrera & 7 & A. P. Russo & 12 & N. Galí & 171 & L. Prats & 0,22307 & I. Cortés & 8 \\
\hline A. Blanco & 7 & M. Villares & 12 & J.F. Valls & 171 & J. F. Valls & 0,22306 & I. Brunet & 8 \\
\hline J. Ò. Guia & 7 & H. Valdemoro & 12 & M. A. Barceló & 158 & J. 0. Sallent & 0,22306 & J. Wilson & 8 \\
\hline L. Cuesta & 7 & L. Cuesta & 12 & J. Blay & 108 & E. Martinez & 0,22304 & J. C. Polo & 7 \\
\hline G. K. Priestley & 7 & R. M. Fraguell & 11 & L. Prats & 103 & J.A. Donaire & 0,22303 & J. Ò. Guia & 7 \\
\hline D. Saurí & 7 & N. Galí & 11 & J.A. Duro & 75 & R. Camprubí & 0,22302 & A. Alarcón & 7 \\
\hline
\end{tabular}

Fuente: elaboración propia. 


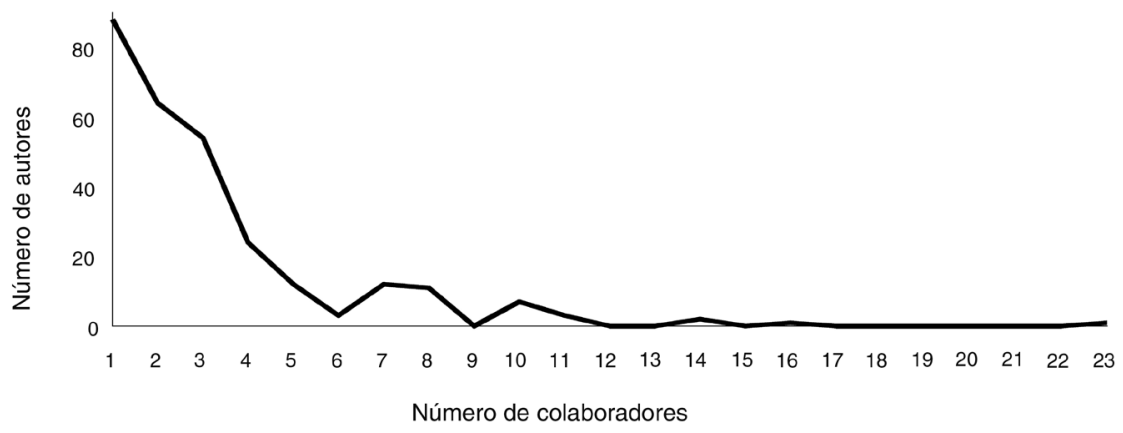

Gráfico 6. Distribución del número de colaboraciones por autor.

Fuente: elaboración propia.

nodos con los que está directamente conectado . Es un indicador del acceso a la información que circula por la red. Para el conjunto de la red, el rango mide su actividad.

El rango medio de la red fue 2,8, o sea, cada autor colaboró con 2,8 colegas en promedio. La distribución del rango fue muy desigual (Gráfico 6). El 85,5\% de los autores tuvo menos de cinco colaboradores, mientras que sólo el 3,9\% tuvo 10 o más. El investigador con más colaboradores fue José Antonio Quintana, con 23. La media de colaboraciones por autor fue 3,4 , es decir, cada autor participó en 3,4 artículos escritos en coautoría en promedio.

La intermediación mide las veces que un nodo aparece en el camino más corto que conecta otros dos nodos. Es una medida del control de información. Informa de la capacidad de una persona de ejercer de intermediaria entre personas que no están conectadas directamente. «Las personas puente son imprescindibles para la vida social, al permitir la conexión de grupos que de otra forma permanecerían aislados» (Molina, 2001: 79). La intermediación media de la red fue de 13,844 .

El grado de cercanía de un nodo indica la capacidad que tiene de alcanzar al resto de nodos de la red. La cercanía de un nodo se ve afectada no sólo por su cantidad de conexiones, sino también por la cercanía de los nodos con los que está conectado. "Una persona poco conectada con el resto (baja centralidad, bajo grado de intermediación), por el solo hecho de estar conectada con una persona "importante", puede tener una alta cercanía y, por tanto, trasladado a la arena del juego político, una gran influencia» (Molina, 2001: 80). La cercanía normalizada media de la red fue de 0,203.

5. Este es el concepto de rango cuando la red es binaria, es decir, cuando los vínculos entre nodos no están valorados. En la red de coautorías, corresponde al número de colaboradores de un autor. Cuando los vínculos están valorados, el rango de un nodo es la suma de los valores de sus vínculos directos con otros nodos. En la red de coautorías, corresponde al número de colaboraciones de un autor. 


\subsection{Colaboración institucional y territorial}

El 69\% de los artículos fueron firmados por autores afiliados a una sola institución; el $25,2 \%$, por dos, y el 5,8\%, por tres o más instituciones. Es decir, solo hubo colaboración institucional en el $31 \%$ de los artículos.

El gráfico 7 muestra la red de colaboración entre las universidades catalanas y otras instituciones. Se consideró que dos instituciones colaboraron si dos de sus afiliados publicaron un artículo en coautoría. Los nodos representan las 116 instituciones colaboradoras y los vínculos, colaboraciones entre instituciones. El tamaño de los nodos es proporcional al número de artículos y el grosor de los vínculos es proporcional al número de colaboraciones. Sólo se indica el nombre de las instituciones con más de tres artículos.

La tabla 4 muestra tres medidas de centralidad de las instituciones con 10 o más artículos: el rango de la red dicotomizada, es decir, el número de instituciones diferentes con las que colaboró cada institución; el rango de la red valorizada, o sea, el número de colaboraciones de cada institución, yla intermediación, esto es, las veces que cada institución aparece en el camino más corto que conecta otras dos instituciones.

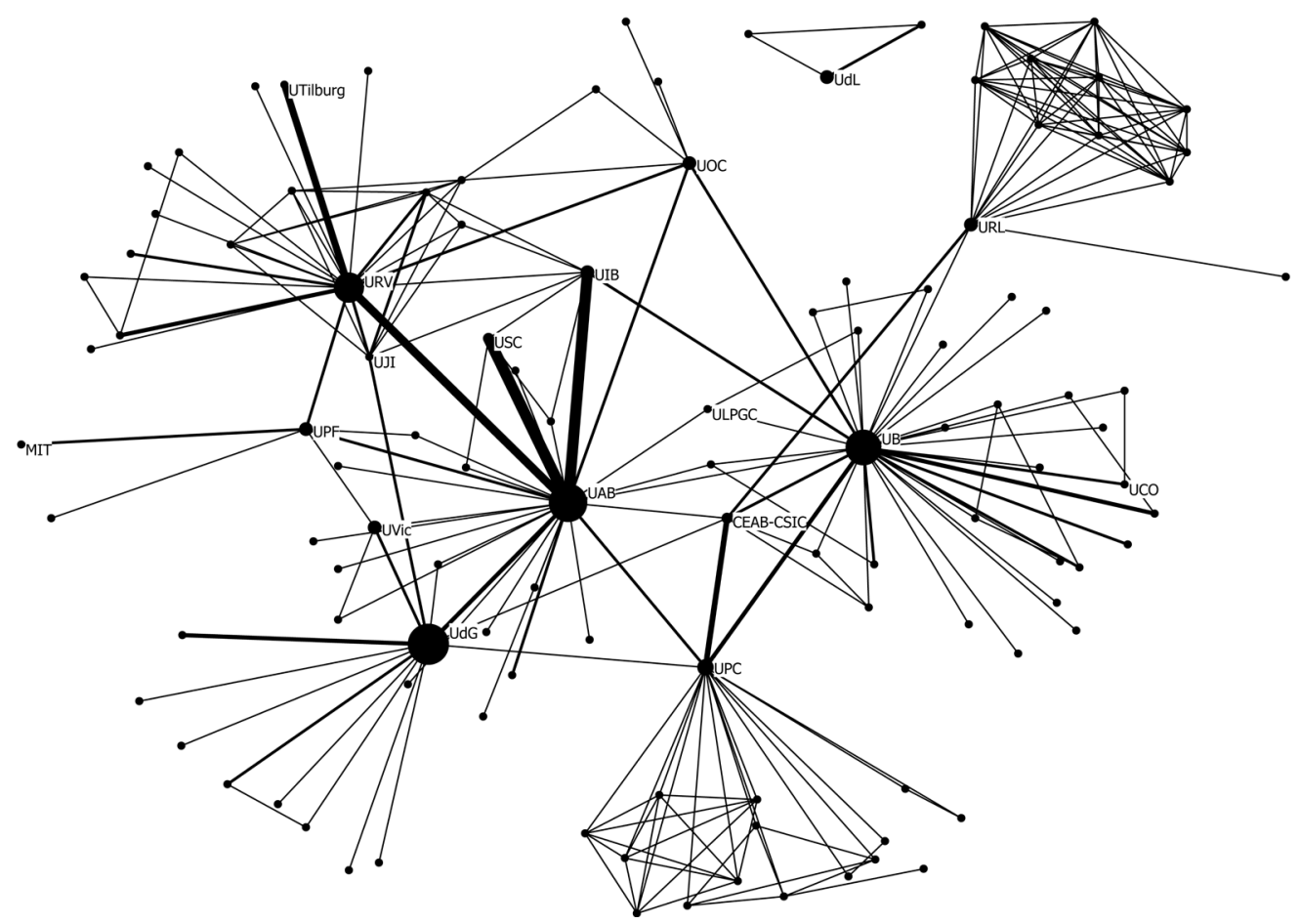

Gráfico 7. Red de colaboración entre las universidades catalanas y otras instituciones.

Fuente: elaboración propia. 
El gráfico 8 muestra la red de colaboración entre Cataluña y otras comunidades autónomas y países. Los nodos representan las 12 comunidades autónomas y los 27 países con los que Cataluña colaboró. El grosor de los vínculos es proporcional al número de colaboraciones. Se consideró que Cataluña colaboró con otra comunidad autónoma o país cuando un autor afiliado a una univer-

Tabla 4. Medidas de centralidad de las instituciones con 10 o más artículos

\begin{tabular}{lcccc}
\hline Institución & Colaboradoras & Colaboraciones & Intermediación & Artículos \\
\hline UB & 34 & 45 & 5.292 & 129 \\
UAB & 27 & 52 & 5.014 & 136 \\
\hline URV & 20 & 36 & 2.415 & 105 \\
UPC & 18 & 24 & 3.105 & 41 \\
UdG & 14 & 21 & 1.879 & 151 \\
URL & 13 & 14 & 2.165 & 29 \\
UIB & 8 & 16 & 661 & 20 \\
UOC & 7 & 10 & 971 & 31 \\
\hline CEAB-CSIC & 7 & 12 & 1.820 & 12 \\
UPF & 6 & 9 & 458 & 27 \\
UVic & 4 & 5 & 40 & 21 \\
USC & 4 & 11 & 4 & 10 \\
UdL & 2 & 3 & 0 & 22 \\
\hline
\end{tabular}

Fuente: elaboración propia.

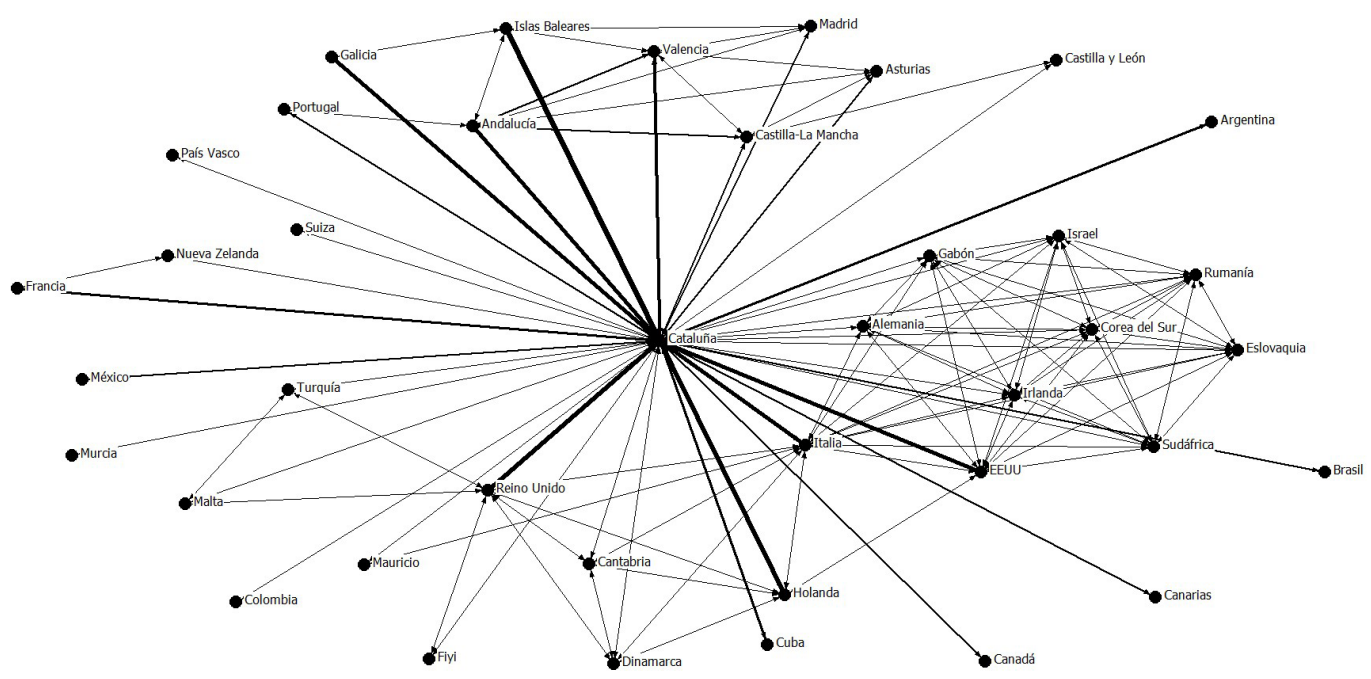

Gráfico 8. Red de colaboración entre Cataluña y otras comunidades autónomas y países.

Fuente: elaboración propia. 
Tabla 5. Distribución de las colaboraciones según niveles*

\begin{tabular}{|c|c|c|}
\hline Nivel de colaboración & Núm. de artículos & $\%$ de artículos \\
\hline Sin colaboración: un único autor & 172 & 40,1 \\
\hline Colaboración local: varios autores de la misma institución & 124 & 28,9 \\
\hline $\begin{array}{l}\text { Colaboración catalana: autores de diferentes instituciones } \\
\text { de Cataluña }\end{array}$ & 47 & 11,0 \\
\hline $\begin{array}{l}\text { Colaboración española: autores de instituciones de Cataluña } \\
\text { y de España }\end{array}$ & 35 & 8,2 \\
\hline $\begin{array}{l}\text { Colaboración internacional: autores de instituciones } \\
\text { de diferentes países }\end{array}$ & 51 & 11,9 \\
\hline Total & 429 & 100,0 \\
\hline
\end{tabular}

sidad catalana publicó un artículo en coautoría con un autor afiliado a una universidad de otra comunidad autónoma o país.

Las cuatro comunidades autónomas con las que Cataluña colaboró más fueron las Islas Baleares $\left(24 \%^{6}\right)$, Galicia (18\%), Andalucía (16\%) y la Comunidad Valenciana (13,3\%). Además, Cataluña colaboró con otras ocho comunidades autónomas (porcentajes inferiores al 10\%). Los cuatro países con los que Cataluña colaboró más fueron el Reino Unido $(14 \% 7)$, Holanda (13\%), Italia (11\%) y Estados Unidos (10\%). Adicionalmente, Cataluña colaboró, pero menos, con otros 23 países.

La colaboración científica puede clasificarse en tres niveles: local, doméstica e internacional (Ardanuy, 2012). En la colaboración local, todos los investigadores trabajan en la misma institución; en la doméstica, participan investigadores de diferentes instituciones de un mismo país, y en la internacional, participan investigadores de distintos países. El 48,2\% de las colaboraciones fueron locales (tabla 5).

Respecto a la evolución de los tres niveles de colaboración, comparando el primer lustro con el segundo, la colaboración que más creció fue la doméstica, que casi se triplicó, mientras que la internacional se duplicó, aproximadamente. La colaboración local, a pesar de ser la más frecuente, fue la que menos aumentó, puesto que se multiplicó por 1,5. El gráfico 9 muestra la evolución anual del porcentaje que representa el número de colaboraciones de cada nivel con respecto al número total de colaboraciones.

6. Este porcentaje se refiere a la proporción que representan las colaboraciones (de Cataluña) con la comunidad autónoma en cuestión, respecto al total de colaboraciones con comunidades autónomas españolas.

7. Este porcentaje se refiere a la proporción que representan las colaboraciones (de Cataluña) con el país en cuestión, respecto al total de colaboraciones con países de fuera de España. 


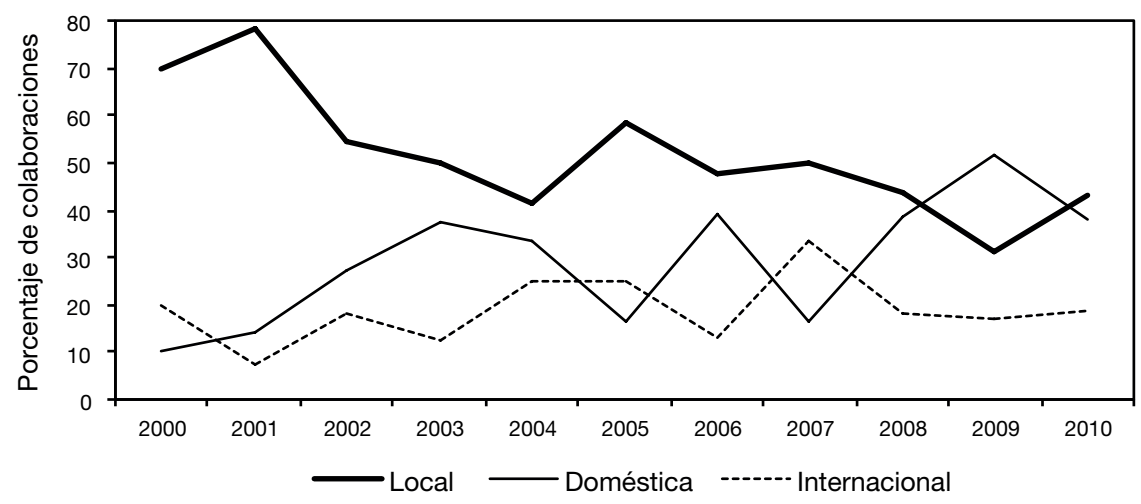

Gráfico 9. Evolución de los tres tipos de colaboración.

Fuente: elaboración propia.

\subsection{Grupos de investigación turistica}

La elaboración del catálogo de grupos de investigación dio como resultado los 20 grupos con dedicación al turismo que se muestran en la tabla 6 . Aunque hubo grupos adscritos a ocho universidades, la UdG y la URV fueron las que albergaron más grupos, cinco cada una. La tabla 7 muestra características de los grupos. Doce grupos fueron reconocidos por la Generalidad de Cataluña en la convocatoria de ayudas para dar apoyo a las actividades de los grupos de investigación de Cataluña (SGR 2009); ocho en la modalidad de grupo consolidado (GRC) y cuatro de grupo emergente (GRE).

El tamaño osciló entre los cinco miembros del grupo más pequeño (LNT) y los 57 del más grande (AQR-IREA). La media fue de 18,7 miembros por grupo y la desviación típica, de 12,2. Respecto al sexo de los investigadores, ECGESTUR fue el grupo con el menor porcentaje de hombres $(37,5 \%)$ y ACE-TCT con el mayor $(77,8 \%)$. El conjunto de los 20 grupos lo formaron 375 personas, el 60,3\% hombres y el 39,7\% mujeres. Y en cuanto a los investigadores principales, 13 grupos estuvieron liderados por hombres y siete por mujeres.

Según las disciplinas científicas asignadas a los grupos en la convocatoria SGR 2009 (ámbito CIRIT ${ }^{8}$ y área de conocimiento), la economía (ocho grupos) y la geografía (seis) fueron las disciplinas predominantes. Dentro de la economía, hubo cinco grupos orientados hacia la economía aplicada y tres hacia la organización de empresas. Además, hubo dos grupos de historia del arte, uno de sociología, uno de comunicación, uno de informática y uno multidisciplinar.

8. Consell Interdepartamental de Recerca i Innovació Tecnològica (en español: Consejo Interdepartamental de Investigación e Innovación Tecnológica). 


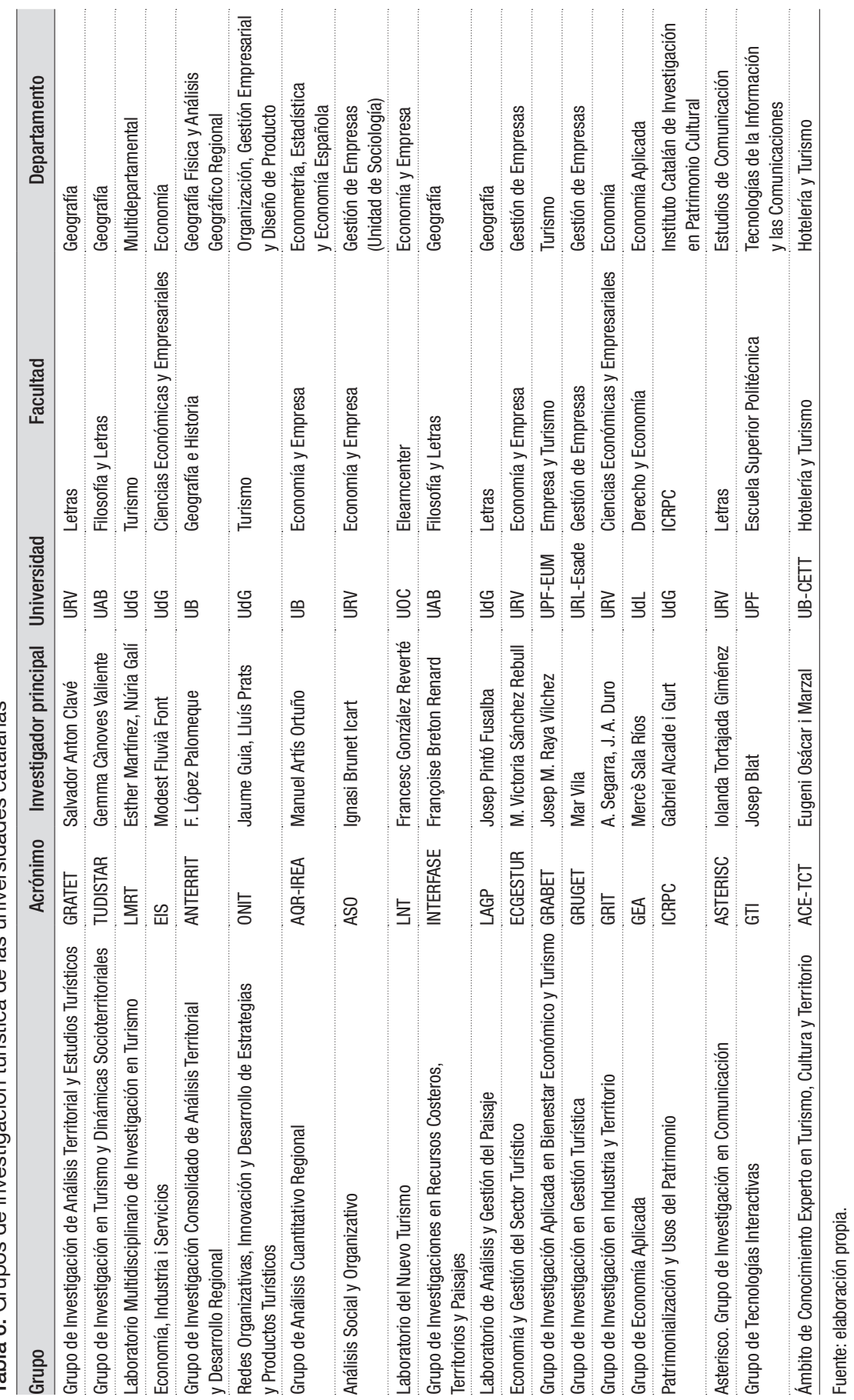




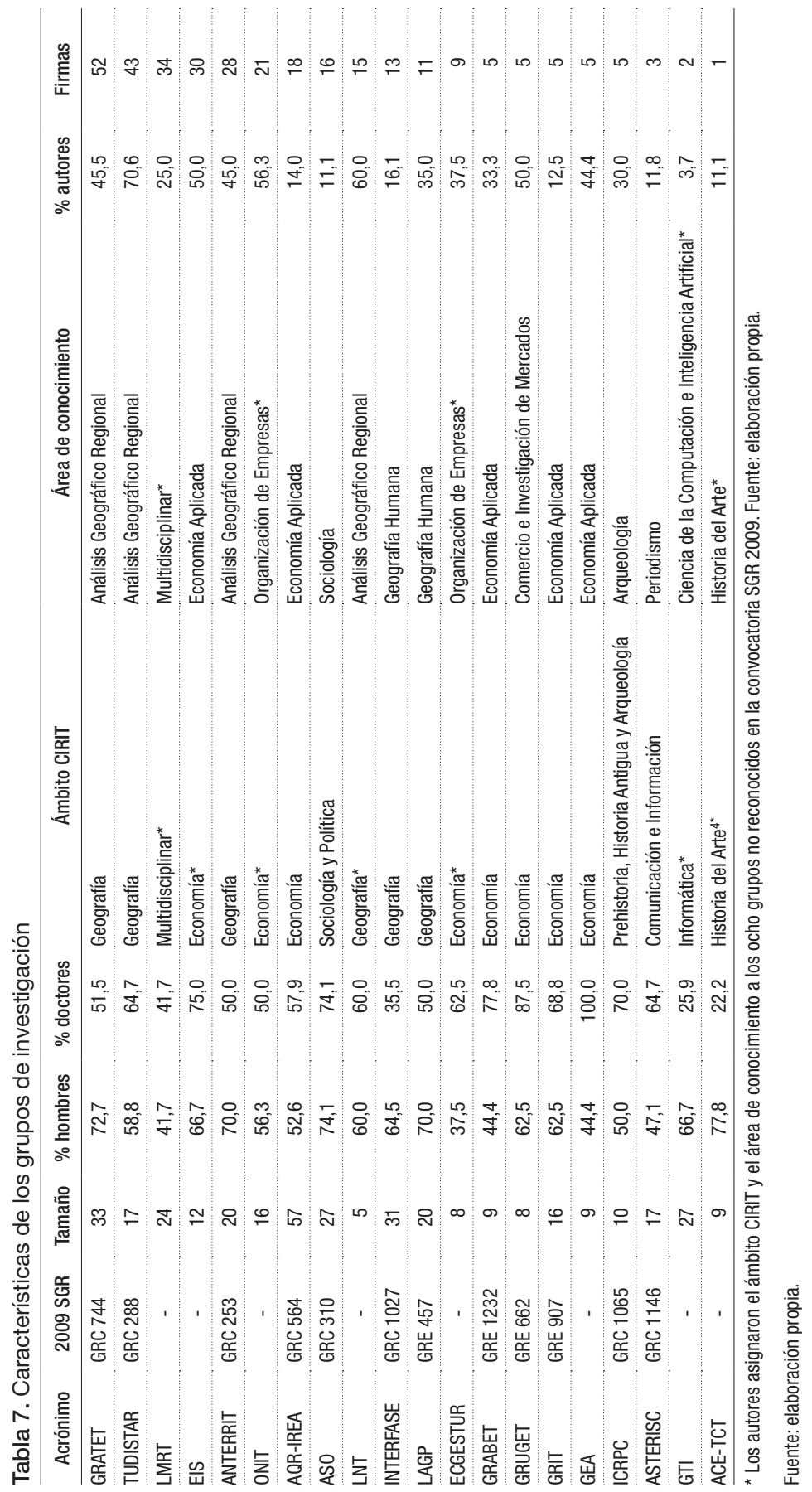


La tabla 7 muestra el porcentaje de miembros que publicaron algún artículo sobre turismo entre 2000 y 2010, de los 429 recopilados en este estudio. Puede considerarse un indicador del grado de dedicación de los grupos al turismo. El rango osciló entre el 70,6\% de TUDISTAR y el 3,7\% del GTI. La media de los 20 grupos fue del $33,1 \%$.

El número de firmas de los grupos en artículos publicados entre 2000 y 2010 puede considerarse un indicador de la producción de los grupos. Miembros de los 20 grupos estamparon 321 de las 884 firmas de los 429 artículos. Es decir, el 36,3\% de ellas fueron de autores pertenecientes a los 20 grupos. La media fue de 16,1 firmas por grupo y la desviación típica, de 14,5 . Y, puesto que 694 de las 884 firmas fueron de autores afiliados a universidades catalanas, los grupos fueron autores del $46,3 \%$ de la producción catalana.

\subsection{Colaboraciones de los grupos de investigación}

La tabla 8 muestra el número de colaboraciones de los grupos. El porcentaje de colaboración interna indica la proporción de colaboraciones entre miembros de un grupo respecto al total de colaboraciones del grupo. En grupos, como

Tabla 8. Colaboraciones internas y externas de los grupos de investigación

\begin{tabular}{lccc}
\hline Grupo & Colaboraciones & $\begin{array}{c}\% \text { de colaboración } \\
\text { interna }\end{array}$ & $\begin{array}{c}\text { \% de colaboración } \\
\text { externa }\end{array}$ \\
\hline ACE-TCT & 0 & 0,0 & 0,0 \\
\hline ASO & 16 & 100,0 & 0,0 \\
\hline ASTERISC & 6 & 33,3 & 66,7 \\
\hline ECGESTUR & 10 & 40,0 & 60,0 \\
\hline EIS & 63 & 50,8 & 49,2 \\
\hline GRABET & 6 & 0,0 & 100,0 \\
\hline ANTERRIT & 18 & 77,8 & 22,2 \\
GRATET & 57 & 24,6 & 75,4 \\
\hline GRUGET & 7 & 85,7 & 14,3 \\
\hline GRIT & 4 & 50,0 & 50,0 \\
\hline TUDISTAR & 104 & 78,8 & 21,2 \\
\hline INTERFASE & 22 & 54,5 & 45,5 \\
\hline GTI & 7 & 0,0 & 100,0 \\
\hline GEA & 5 & 40,0 & 60,0 \\
\hline LAGP & 37 & 27,0 & 73,0 \\
LNT & 13 & 61,5 & 38,5 \\
\hline LMRT & 32 & 56,3 & 43,8 \\
\hline ICRPC & 5 & 40,0 & 60,0 \\
\hline AQR-IREA & 30 & 40,0 & 60,0 \\
\hline ONIT & 41 & 63,4 & 36,6 \\
\hline FUE & & &
\end{tabular}

Fuente: elaboración propia. 


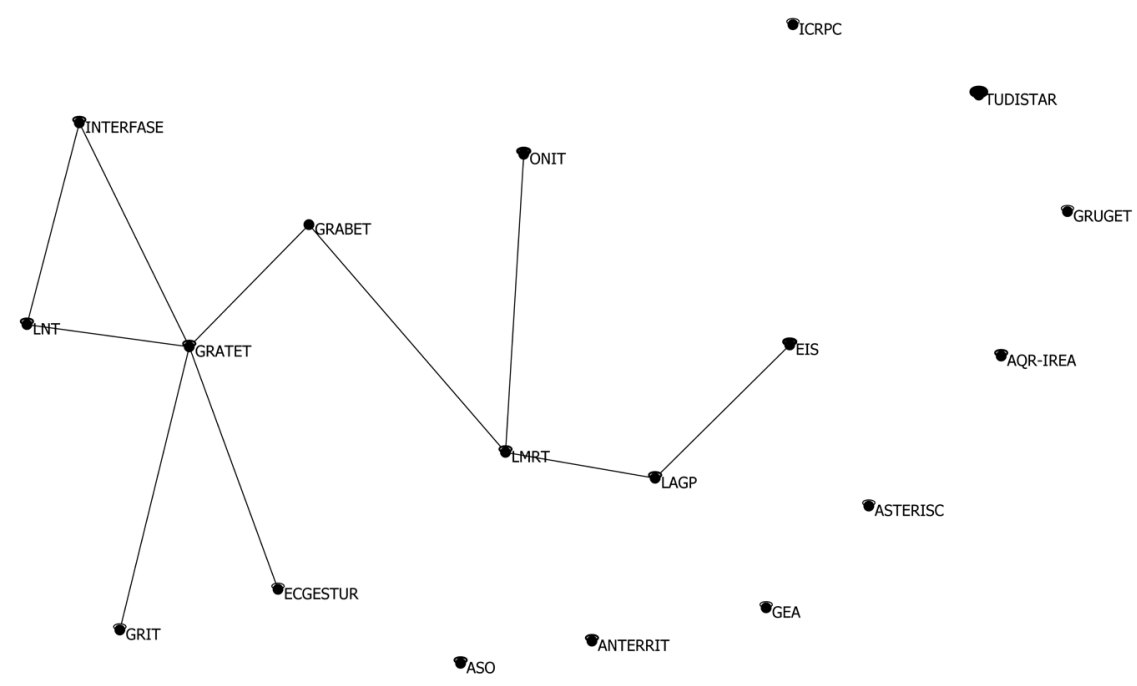

Gráfico 10. Red de colaboración de los grupos de investigación.

Fuente: elaboración propia.

TUDISTAR, predominó la colaboración interna frente a la externa, mientras que en otros, como GRATET, predominó la colaboración con investigadores de fuera del grupo. Los promedios de colaboración interna y externa fueron el $54,2 \%$ y el $45,8 \%$, respectivamente.

El 19,9\% de las colaboraciones externas fue entre los 20 grupos, mientras que el $80,1 \%$ fue con investigadores no pertenecientes a los 20 grupos. Y hubo 11 investigadores pertenecientes a dos grupos al mismo tiempo. El gráfico 10 muestra la red de colaboración de los 20 grupos. Los nodos representan grupos, y los vínculos, colaboraciones. El grosor de los vínculos es proporcional al número de colaboraciones. Los bucles representan colaboraciones internas?.

\section{Discusión y conclusiones}

La incidencia de la coautoría es similar a la medida en otros estudios (tabla 9) y el aumento moderado de la incidencia es congruente con la tendencia general en la investigación científica (Sonnenwald, 2007). La extensión de la coautoría es mayor que en los estudios de Benckendorff (2010) y Ye et al. (2011), en el campo del turismo, y Acedo et al. (2006), en el campo de la administración.

9. El gráfico 10 muestra las colaboraciones internas de los 20 grupos y las colaboraciones externas entre los 20 grupos, sin embargo, no muestra las colaboraciones externas con investigadores no pertenecientes a los 20 grupos. 
Tabla 9. Comparación de medidas de colaboración en varios estudios

\begin{tabular}{|c|c|c|c|c|c|c|}
\hline & Este estudio & $\begin{array}{c}\text { Benckendorff } \\
\text { (2010) }\end{array}$ & $\begin{array}{l}\text { Ye et al. } \\
(2011)\end{array}$ & $\begin{array}{l}\text { Acedo et al. } \\
(2006)\end{array}$ & $\begin{array}{c}\text { Newman } \\
(2001)\end{array}$ & $\begin{array}{c}\text { Newman } \\
(2001)\end{array}$ \\
\hline Campo & Turismo & Turismo & $\begin{array}{l}\text { Turismo y } \\
\text { hostelería }\end{array}$ & Administración & Biomedicina & $\begin{array}{l}\text { Física de } \\
\text { partículas }\end{array}$ \\
\hline Ámbito & Cataluña & $\begin{array}{l}\text { Australia y } \\
\text { Nueva Zelanda }\end{array}$ & General & General & General & General \\
\hline Revistas & $\begin{array}{l}\text { Turísticas y } \\
\text { no turísticas }\end{array}$ & 18 turísticas & $\begin{array}{l}6 \text { de turismo } \\
\text { y hostelería }\end{array}$ & $\begin{array}{l}10 \text { de } \\
\text { administración }\end{array}$ & MEDLINE & SPIRES \\
\hline Periodo & $2000-2010$ & $1999-2008$ & $1991-2010$ & $1980-2002$ & 1995-1999 & $1995-1999$ \\
\hline Incidencia & 59,9 & 61,58 & 59,51 & n. d. & n. d. & n. d. \\
\hline Extensión & 2,77 & 1,9 & 1,87 & 1,88 & 3,75 & 8,96 \\
\hline Colaboradores por autor & 2,807 & n. d. & 2,58 & 2,43 & 18,1 & 173 \\
\hline$\%$ componente principal & 12,01 & 29,19 & 59,3 & 45,45 & 92,6 & 88,7 \\
\hline Densidad & 0,0055 & 0,003 & n. d. & 0,0002 & n. d. & n. d. \\
\hline
\end{tabular}

Fuente: elaboración propia a partir de Benckendorff (2010), Ye et al. (2011), Acedo et al. (2006) y Newman (2001).

Y es menor que la obtenida por Newman (2001) en los campos de la biomedicina y la física de partículas. Se esperaba una menor extensión de la coautoría que en las ciencias naturales y experimentales, pero no se esperaba que fuera mayor que en otros estudios en el campo del turismo. Una explicación es la distorsión que hayan podido causar seis artículos con un número infrecuente de autores (dos artículos con ocho autores, dos con nueve y dos con once), en una muestra comparativamente reducida.

En general, cuatro autores parecen representar el techo al número de autores por artículo. Los resultados corroboran la reticencia que existe en las ciencias sociales a que un alto número de autores firmen los trabajos (Acedo et al., 2006). Si el estudio hubiera incluido la investigación turística en biomedicina, como la medicina del viajero, probablemente la extensión de la coautoría hubiera resultado mayor. Una posible explicación del límite de autores por trabajo pueden ser los criterios de evaluación de los investigadores (Durà et al., 2007). Las agencias evaluadoras de Cataluña (AQU) y España (ANECA) tienen en cuenta el número de autores al valorar las publicaciones científicas.

Las diferencias entre revistas en la incidencia y la extensión de la coautoría parecen indicar que el tipo de revista influye en la colaboración. La mayor colaboración en las revistas más prestigiosas —aquéllas indexadas en ISI y Scopus - puede explicarse por su mayor exigencia de calidad (Acedo et al., 2006) y quizás también por la dificultad del idioma para los autores catalanes, ya que la mayoría son en inglés. Ante una mayor dificultad percibida, los autores aúnan esfuerzos para aumentar la probabilidad de que su artículo sea aceptado.

Por otra parte, los resultados no corroboran la asunción de que la multidisplinariedad aumente la colaboración. En el caso de las revistas españolas, el efecto de la colaboración sobre la incidencia es contrario al esperado: disminuye 
la colaboración cuando aumenta el número de disciplinas. Quizás se hayan escogido indicadores inapropiados para medir la multidisciplinariedad, y la asignación de disciplinas por la indexación de ISOC no capte bien la multidisciplinariedad. En el caso de las revistas indexadas en Scopus, los resultados sí encajan con lo esperado, pues tanto la incidencia como la extensión de la colaboración aumentan con el número de disciplinas, sin embargo, los resultados no son estadísticamente significativos.

La red de coautorías está poco cohesionada: hay multitud de investigadores aislados y subgrupos desconectados. La densidad puede considerarse muy baja, sobre todo teniendo en cuenta que los investigadores se encuentran en un territorio relativamente pequeño y, por tanto, a corta distancia unos de otros. Y el tamaño del componente principal es muy pequeño (tabla 9). La gran fragmentación de la red social y el escaso desarrollo del componente principal pueden deberse a la inmadurez de la investigación turística y a su naturaleza multidisciplinar (Racherla y Hu, 2010).

Las medidas de centralidad de los nodos de la red de coautorías ponen de manifiesto cuáles son los investigadores clave de las universidades catalanas. Se observa que los autores más productivos no son siempre los más centrales, por ejemplo: Gemma Cànoves, Núria Galí, Josep Maria Espinet, Belén Gómez, etc. son investigadores prolíficos en turismo, pero con bajo grado de intermediación. Se trata de estudiosos que colaboran frecuentemente con un reducido número de colegas, en subgrupos relativamente aislados. Otros autores prolíficos sí ocupan una posición central en la red, como Modest Fluvià y Salvador Anton.

Los considerables aumentos de la colaboración nacional e internacional pueden explicarse por las mejoras en los sistemas de comunicación y por los criterios de las agencias evaluadoras, por ejemplo: un criterio transversal de valoración de la convocatoria de ayudas para dar apoyo a las actividades de los grupos de investigación de la AGAUR (SGR 2009) es el grado de internacionalización de las actividades de investigación del grupo.

Para esbozar una tipología tentativa de grupos, un primer criterio que los discrimina es el grado de dedicación al turismo. Hay dos clases de grupos: los que tienen el turismo como objeto de estudio central y los que no, lo cual se refleja en las publicaciones, la misión y las líneas de investigación. El segundo criterio es la principal orientación disciplinar: geografía, economía aplicada, organización de empresas y «otras disciplinas». El tercer criterio es el tamaño del grupo: pequeño o grande.

El primer tipo consiste en grupos grandes, con alta dedicación al turismo y orientados a la geografía. Se trata de GRATET, ANTERRIT, TUDISTAR y LAGP. Los grupos LMRT y LNT son similares a este tipo; aunque entre las disciplinas científicas desde las que investiga el LMRT se encuentran, además de la geografía, la economía y la historia del arte, y el LNT es un grupo pequeño. El segundo tipo lo forman grupos pequeños, con alta dedicación al turismo y orientados hacia la economía aplicada. Son EIS, GEA y GRABET. El tercer tipo está compuesto por grupos con alta dedicación al turismo y orientados 
hacia la organización de empresas. Se trata de ONIT, ECGESTUR y GRUGET (aunque ONIT es mayor que los otros dos). Y el cuarto tipo consiste en grupos con baja dedicación al turismo dentro del conjunto de su actividad. Lo forman el resto de grupos y es heterogéneo en cuanto a tamaños y enfoques disciplinares.

Los 20 grupos producen casi la mitad de los artículos sobre turismo de las universidades catalanas, y autores no pertenecientes a los 20 grupos producen la otra mitad. Esto plantea una situación ambivalente. Los grupos cumplen una función básica en la creación de conocimiento turístico, aunque también es sustancial la aportación de investigadores no pertenecientes a los 20 grupos.

Por otra parte, los grupos son algo más que unidades estructurales (Cohen, 1991), porque realmente colaboran internamente. Pero que el $45,8 \%$ de las colaboraciones sean externas indica que, al mismo tiempo, no son unidades cerradas y que su grado de funcionalidad no es total.

Con respecto a las colaboraciones externas de los grupos, sólo una quinta parte tiene lugar entre los 20 grupos. Y la mitad de ellos no han colaborado nunca con otro grupo de investigación turística de Cataluña. Quizás una mayor colaboración entre los grupos les permitiría conseguir sinergias positivas. Las colaboraciones entre ellos se han producido sobre todo entre los de una misma universidad: entre grupos de la UdG, por un lado, y entre grupos de la URV, por el otro. Parece que la proximidad geográfica favorece la colaboración entre grupos.

\section{Bibliografía}

Acedo, Francisco J.; Barroso, Carmen; Casanueva, Cristobal y Galán, José L. (2006). «Co-Authorship in Management and Organizational Studies: An Empirical and Network Analysis». Journal of Management Studies, 43 (5), 957-983.

AlbaCETE, Carlos A. y FuENTES, María del Mar (2010). «Difusión de la investigación española sobre turismo en revistas internacionales». Revista de Análisis Turistico, 9, 14-29.

ARDANUY, Jordi (2012). «Scientific collaboration in Library and Information Science viewed through the Web of Knowledge: the Spanish case». Scientometrics, 90 (3), 877-890.

Barriocanal, Carles; Crous, Anna; VARGa, Diego y Vila, Josep (2007). «Anàlisi de les revistes de geografia indexades al Journal of Citation Reports del Social Science Citation Index. Període 1997-2005». Documents d'Anàlisi Geogràfica, 50, 51-72.

Barrios, Maite; Borrego, Ángel; Vilaginés, Andreu; Ollé, Candela y Somoza, Marta (2008). "A bibliometric study of psychological research on tourism». Scientometrics, 77 (3), 453-467.

BEAVER, Donald de B. (2001). «Reflections on scientific collaborations (and its study): past, present and future». Scientometrics, 52 (3), 365-377.

BENCKENDORFF, Pierre (2009). «Themes and trends in australian and new zealand tourism research: A social network analysis of citations in two leading journals (1994-2007)». Journal of Hospitality and Tourism Management, 16 (1), 1-15.

- (2010). «Exploring the limits of tourism research collaboration: A social network analysis of co-authorship patterns in Australian and New Zealand tourism research». Proceedings of the 20th Annual CAUTHE Conference. Hobart, Tasmania. 
Ceballos, Cristina; Arias, Carlos; Ruiz, Antonio; Sanz, Carlos y VÁzQuez, Isabel (2010). «La formación en turismo en España: pasado, presente y futuro en el nuevo Espacio Europeo de Educación Superior». Cuadernos de Turismo, 25, 45-67.

Chang, Chia-Lin y MCAleER, Michael (2012). «Citations and impact of ISI tourism and hospitality journals». Tourism Management Perspectives, 1, 2-8.

CoHEN, J. E. (1991). «Size, age and productivity of scientific and technical research groups». Scientometrics, 20 (3), 395-416.

Corral, José A. y CÀnOves, Gemma (2013). «La investigación turística publicada en revistas turísticas y no turísticas: Análisis bibliométrico de la producción de las universidades catalanas». Cuadernos de Turismo, 31, 55-81.

De Solla Price, Derek J. (1966). Little Science, Big Science. Nueva York: Columbia University Press.

Direcció General de Turisme (2011). Catalunya turística en xifres 2010. Barcelona: Generalitat de Catalunya.

Durà, Antoni; Oliveras, Xavier y Villanueva, Maria (2007). «Vint-i-cinc anys de difusió de la recerca geogràfica: Anàlisi bibliomètrica de la revista Documents d'Anàlisi Geogràfica». Documents d'Anàlisi Geogràfica, 50, 29-50.

ETZKOWITZ, Henry (2003). "Research groups as "quasi-firms": The invention of the entrepreneurial university». Research Policy, 32 (1), 109-121.

EUROSTAT (2011). Europe in figures. Eurostat yearbook 2011. Luxemburgo: Eurostat.

FERnÁndeZ, Alfonso; GARCÍA, María e IVARS, Josep A. (coords.) (2010). La investigación de la geografía del turismo en las comunidades autónomas españolas. Madrid: Asociación de Geógrafos Españoles. Grupo de Geografía del Turismo, Ocio y Recreación.

FREEMAN, Linton C. (1978-1979). "Centrality in social networks conceptual clarification». Social Networks, 1 (3), 215-239.

Garay, Luis A. y CANOves, Gemma (2010). "Un análisis del desarrollo turístico en Cataluña a través del ciclo de evolución del destino turístico». Boletín de la Asociación de Geógrafos Españoles, 52, 43-58.

GLÄNZEL, Wolfgang y SCHUBERT, András (2004). "Analysing scientific networks through coauthorship». En: MoED, Henk F.; GLÄNZEL, Wolfgang y SCHMOCH, Ulrich (eds.). Handbook of quantitative science and technology research. The Netherlands: Springer, 257-276.

González-Albo, Borja; Moreno, luz; Aparicio, Javier; Morillo, Fernanda y Bordons, María (2009). La investigación española sobre turismo a través de publicaciones científicas de difusión nacional e internacional. IEDCYT, CCHS, CSIC. Documento no publicado.

HernándeZ, José M.; CAMPÓn, Ana M. y Folgado, José A. (2011). «La investigación en turismo en España a través de las revistas de dirección de empresas». Revista de Análisis Turístico, 12, 1-9.

KATZ, J. Sylvan y MARTIN, Ben R. (1997). "What is research collaboration?». Research Policy, 26 (1), 1-18.

LABAND, David N. y TOlLISON, Robert D. (2000). «Intellectual collaboration». Journal of Political Economy, 108 (3), 632-662.

MCKerCHER, Bob; LAW, Rob y LAM, Terry (2006). «Rating tourism and hospitality journals». Tourism Management, 27 (6), 1235-1252.

Molina, José L. (2001). El análisis de redes sociales: Una introducción. Barcelona: Edicions Bellaterra.

- (2004). «La ciencia de las redes». Apuntes de Ciencia y Tecnología, 11, 36-42. 
Moreno, Sergio y PICAzo, Patricia (2012). «Difusión de la investigación científica en revistas de turismo realizada por instituciones españolas». Revista de Análisis Turistico, 14, 33-52.

Newman, Mark E. J. (2001). "The structure of scientific collaboration networks». Proceedings of the National Academy of Sciences, 98 (2), 404-409.

RaCHerla, Pradeep y Hu, Clark (2010). "A social network perspective of tourism research collaborations». Annals of Tourism Research, 37 (4), 1012-1034.

ReY, Jesús; MARTín, M. José y SEBASTIÁN, Jesús (2008). «Estructura y dinámica de los grupos de investigación». Arbor. Ciencia, Pensamiento y Cultura, 184 (732), 743-757.

SÁnCHEZ, Manuel y MARÍN, M. Belén (2003). "La investigación en turismo y economía de la empresa publicada en revistas especializadas españolas: 1996-2001». Papers de Turisme, 33, 6-39.

SANCHO, Amparo (2006). [Sin título.] En: Pulido, Juan I. (coord.). Debate: Validez de las fuentes de información del turismo español. Revista de Análisis Turístico, 2, 57-58.

Severt, Denver E.; Tesone, Dana V.; BotTorff, Timothy J. y Carpenter, Monica L. (2009). "A world ranking of the top 100 hospitality and tourism programs». Journal of Hospitality \& Tourism Research, 33, 451-470.

Sonnenwald, Diane H. (2007). "Scientific collaboration». Annual Review of Information Science and Technology, 41 (1), 643-681.

TORRES, Anna (2010). «Sostenibilitat i modalitats turístiques: Una anàlisi de casos a Catalunya». Documents d'Anàlisi Geogràfica, 56 (3), 479-502.

XiAO, Honggen y SMITH, Stephen L. J. (2006). "The making of tourism research: Insights from a social sciences journal». Annals of Tourism Research, 33 (2), 490507.

YE, Qiang; LI, Tong y LAw, Rob (2013). «A coauthorship network analysis of tourism and hospitality research collaboration». Journal of Hospitality \& Tourism Research, 37 (1), 51-76.

ZHAO, Weibing y RitCHIE, J. R. Brent (2007). «An investigation of academic leadership in tourism research: 1985-2004». Tourism Management, 28 (2), 476-490. 